\title{
Modelo de Comportamiento de Conductores de Automóviles Usando Razonamiento Basado en Contextos
}

\author{
M.Sc. Maureen Murillo ${ }^{1}$ \\ ${ }^{1}$ Escuela de Ciencias de la Computación e Informática, Facultad de Ingeniería, Universidad de \\ Costa Rica, Costa Rica \\ maureenm@cariari.ucr.ac.cr
}

\begin{abstract}
Resumen - En este artículo se exponen los resultados de un proyecto de investigación en el área de Inteligencia Artificial, específicamente en el campo de la representación del conocimiento humano. Se presenta un modelo conceptual o teórico del comportamiento de conductores de automóviles, cuyo desarrollo está basado en el paradigma de representación llamado Razonamiento Basado en Contextos (CxBR - Contextbased Reasoning). El modelo está basado en el perfil de un conductor de Costa Rica con ciertas características, constituyéndose así en un modelo ejemplo para desarrollar futuras simulaciones de tráfico vehicular en otros países. El artículo presenta una descripción del paradigma de Razonamiento Basado en Contextos y luego expone el modelo conceptual del comportamiento de un conductor.
\end{abstract}

Palabras clave: Razonamiento Basado en Contextos, Comportamiento Humano, Inteligencia Artificial.

Abstract - This article shows the results of a research project in the area of Artificial intelligence, specifically in the field of the representation of the human knowledge. It presents a conceptual or theoretical model of the behavior of automobile drivers, whose development is based on the representation paradigm called Contextbased Reasoning (CxBR). The model is based on the profile of a Costa Rican automobile driver with certain characteristics, constituting itself thus in a model example to develop future simulations of vehicular traffic in other countries. The article presents a description of the paradigm Context-base Reasoning and then it exposes the conceptual model of automobile drivers' behavior.

Key-words: Context-based Reasoning, Human Behavior, Artificial Intelligence.

\section{Introducción}

Conducir adecuadamente un automóvil dentro de un ambiente en el que se desenvuelven entes inteligentes (peatones, otros conductores) y otros no tan inteligentes (perros, vacas) es una tarea compleja. Esto debe sumarse a la interpretación de objetos inanimados como señales de tránsito, obstáculos (huecos, bolas) y situaciones inesperadas (desperfectos mecánicos, accidentes), lo que complica aún más la conducción de un automóvil. El conducir un carro puede variar radicalmente en diferentes culturas, ya que depende, además de las regulaciones locales, de la educación y costumbres de los conductores y de las particularidades del ambiente. Esta es una actividad que requiere de conocimiento táctico para tomar decisiones en tiempo real, por ejemplo en intersecciones de calles, lugar donde se concentran los diferentes elementos del ambiente de conducción.

Los modelos y simuladores de manejo actuales son utilizados tanto en investigación y diseño de vehículos, como en entrenamiento. Sin embargo, la mayoría de estos simuladores carecen de un nivel adecuado de realismo, el cual puede ser incrementado incorporando en el ambiente simulado vehículos autónomos [1]. Es necesario entonces, incorporar en los modelos el comportamiento táctico de los conductores para representar estos vehículos autónomos.

El Razonamiento Basado en Contextos (CxBR) es un paradigma de razonamiento automático que puede simular el comportamiento táctico humano en forma simple y efectiva, utilizando un identificador intuitivo llamado contexto. Un contexto, el cual representa todas las condiciones de una situación particular, controla el comportamiento de la entidad inteligente en una simulación. Cuando la situación cambia, el contexto activo actual busca otro posible contexto que pueda controlar las condiciones de la nueva situación [2]. Los distintos contextos se organizan jerárquicamente.

Así como se desarrolla un sistema experto que incorpora el conocimiento de un experto 
humano específico en un área particular y que a su vez constituye un sistema de conocimiento representativo de los expertos en general del área, en este proyecto se pretende modelar el comportamiento representativo de un tipo definido de conductor de automóviles en Costa Rica, el cual podría ser el de otro país latinoamericano.

\section{Metodología}

El modelo teórico desarrollado corresponde a un conductor con las siguientes características:

- Costarricense

- Hombre

- Entre 35 y 45 años

- Con un mínimo de experiencia en manejo de automóviles de 5 años

Se entrevistaron 4 conductores con las características señaladas anteriormente. De los resultados de las entrevistas se extrajo el comportamiento táctico representativo de los conductores con el perfil mencionado. Dado que los conductores costarricenses se rigen con las mismas leyes de tránsito y su experiencia se ha desarrollado en el mismo ambiente y bajo la misma cultura, se considera que las diferencias existentes entre diferentes conductores con el mismo perfil son pequeñas. Esto, unido a consideraciones prácticas, justifica la cantidad de conductores a entrevistar.

\section{Base teórica}

\section{Razonamiento Basado en Contextos}

Las condiciones actuales de tráfico vehicular, especialmente en Costa Rica, tienen características que dificultan la conducción adecuada de vehículos. Si tomamos en cuenta el volumen de carros en las calles, la agresividad de las personas, un ritmo de vida acelerado y el estado deficiente de la infraestructura vial en algunos (o muchos) casos, entre otros factores, podemos suponer que el descifrar el comportamiento de una persona manejando bajo estas circunstancias no es una tarea trivial. Aún más, el modelar ese comportamiento debe tomar en cuenta bastantes variables, de las cuales muchas son externas al conductor y surgen en el momento mismo de la conducción.

Uno de los componentes esenciales de retroalimentación para el conductor de un vehículo es el tráfico vehicular, el cual comparte el mismo ambiente de conducción. El razonamiento basado en contextos (conocido como Context-based
Reasoning - CxBR) es un paradigma excelente para el modelaje de entes autónomos en un mundo simulado [1]. Este paradigma de modelaje fue propuesto por Avelino González y Robert Ahlers [3] para representar y razonar con comportamiento humano.

Este paradigma se ha utilizado principalmente para simular ambientes en donde interactúan oponentes, por ejemplo submarinos o tanques en batalla. En Costa Rica no existen situaciones de guerra, sin embargo, la conducción actual de vehículos puede compararse a un campo de batalla en donde el objetivo es defenderse de "enemigos" (otros vehículos, huecos, etc.) y llegar al destino "sanos y salvos".

Para desarrollar un modelo siguiendo el razonamiento basado en contextos deben definirse una serie de elementos: la misión, contextos principales, contextos reactivos, subcontextos, funciones y reglas sentinela.

\section{Misión}

Una misión especifica un objetivo, que se espera alcanzar mediante la activación y desactivación de contextos principales y subcontextos. Una misión define tanto los objetivos como las restricciones de la operación [4]. Algunos ejemplos de una misión son los siguientes:

- En el dominio de conducción de automóviles: "Transportarse en automóvil desde la universidad al aeropuerto internacional".

- En el dominio de una batalla con submarinos: "Buscar y destruir submarinos enemigos".

De esta forma, una misión requiere de la planificación de una secuencia de contextos que deberán irse activando y desactivando paulatinamente hasta alcanzar el objetivo deseado.

\section{Contextos}

El paradigma basado en contextos parte del supuesto de que un ser humano no aplica todo su conocimiento al desempeñarse en una situación particular, sino que utiliza un conjunto limitado de ese conocimiento el cual está relacionado con la situación específica. Por ejemplo, un conductor en una autopista típicamente no se preocupa de vehículos estacionados que inicien la maniobra de poner el vehículo marcha atrás. Mientras que en un parqueo el conductor sí está vigilante de un suceso de este tipo. 
Bajo este concepto, con el paradigma CxBR se definen contextos, donde cada uno de ellos representa una situación particular en la cual se desenvuelve un ente inteligente, como parte de la misión. El contexto es el elemento representativo más importante en este paradigma. Se representan como clases que encapsulan funciones para ejecutar ciertos comportamientos y acciones típicas de tales contextos [5].

La misión de un modelo, descrita anteriormente, constituye en sí misma un contexto. A nivel general se identifican tres tipos de contextos: la misión, los contextos principales y los subcontextos.

Los contextos principales contienen toda la información necesaria para operar el ente inteligente bajo las situaciones principales en las que se podría desempeñar. Esta información se representa mediante funciones. También contienen el conocimiento necesario para reconocer cuándo el contexto principal activo debe desactivarse para activar otro [5]. Este conocimiento se representa mediante reglas sentinela (véase figura 1).

\section{Contexto principal}

- Funciones

- Reglas sentinela

Figura 1 - Estructura de un contexto principal

Los contextos principales son excluyentes entre sí, es decir, sólo puede estar activo uno a la vez. Cuando una regla sentinela del contexto activo detecta ciertas condiciones pertenecientes a otro contexto principal, entonces desactiva el actual y activa el adecuado.

En una misión de transportarse en automóvil de una localidad a otra, pueden identificarse contextos tales como: conducir en ciudad, conducir en autopista y conducir en parqueo.

Además de los contextos principales básicos que conllevan al logro de la misión, pueden definirse otros especiales llamados contextos reactivos principales. Estos contextos se definen con el objetivo de que se activen en situaciones que podrían darse excepcionalmente en el transcurso de la misión, pero que bajo condiciones "normales" no se activarían. Algunos ejemplos de estos contextos dentro del dominio de conducción de automóviles son: reparar automóvil y llenar tanque de combustible.

El tercer tipo de contextos (subcontextos) se explicará a continuación.

\section{Subcontextos}

El término subcontexto hace referencia a un contexto que es contenido por otro contexto. Un subcontexto representa el comportamiento en una situación específica y limitada que se da dentro de un contexto mayor. Un subcontexto puede a su vez contener sub-subcontextos, y la composición puede

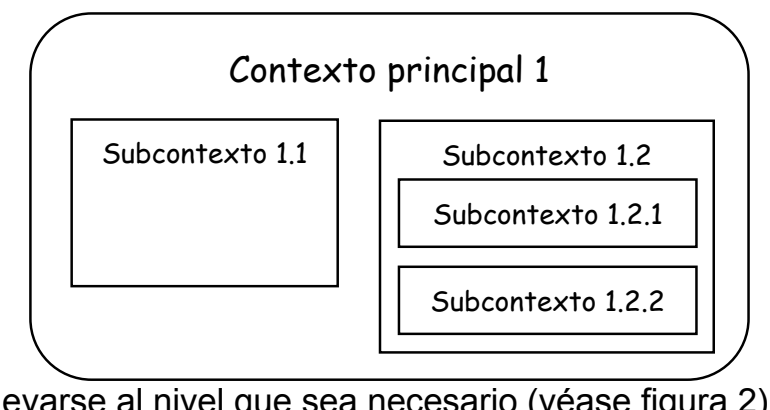

Figura 2- Ejemplo de composición de contextos y subcontextos

La composición de contextos y subcontextos puede plantearse como una jerarquía de contextos. De esta forma, podemos hablar del nivel del contexto, donde los contextos principales son de nivel 1, los subcontextos de los contextos principales son de nivel 2, y así sucesivamente (véase figura 3).

Cada contexto principal se describe como un conjunto de funciones y de reglas sentinela. Las funciones son las actividades que se llevan a cabo dentro de un contexto, mientras que las reglas sentinela vigilan la ocurrencia de eventos que conllevan a la transición hacia otros contextos del mismo nivel o a los subcontextos propios del contexto.

Por otro lado, cada subcontexto puede describirse como una función, o como un conjunto de funciones. Esto depende de la complejidad del subcontexto y de si éste contiene sub-subcontextos. En el caso de que un subcontexto contenga subsubcontextos deberá definir un conjunto de reglas sentinela que le permitan activar los subsubcontextos (véase figura 4). 


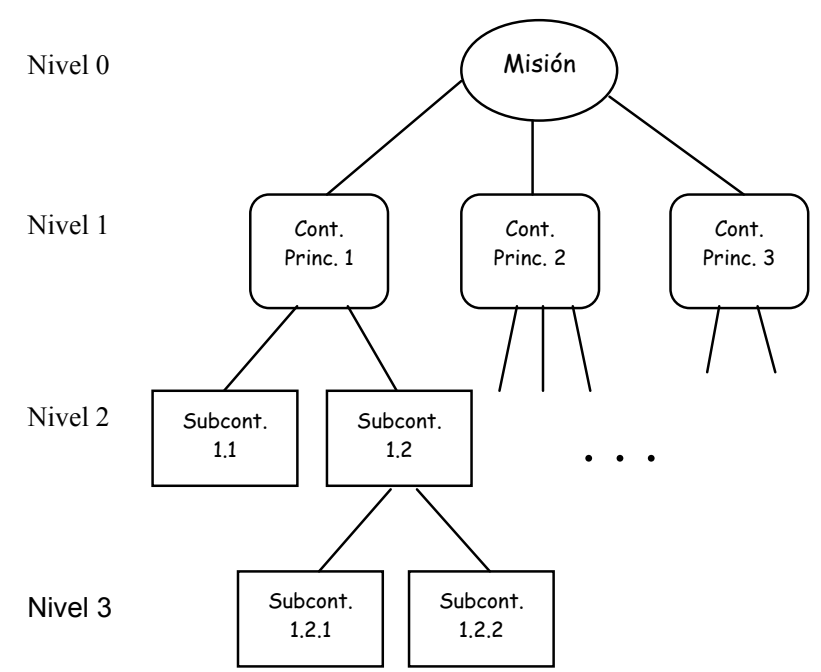

Figura 3 - Ejemplo de jerarquía de contextos y subcontextos

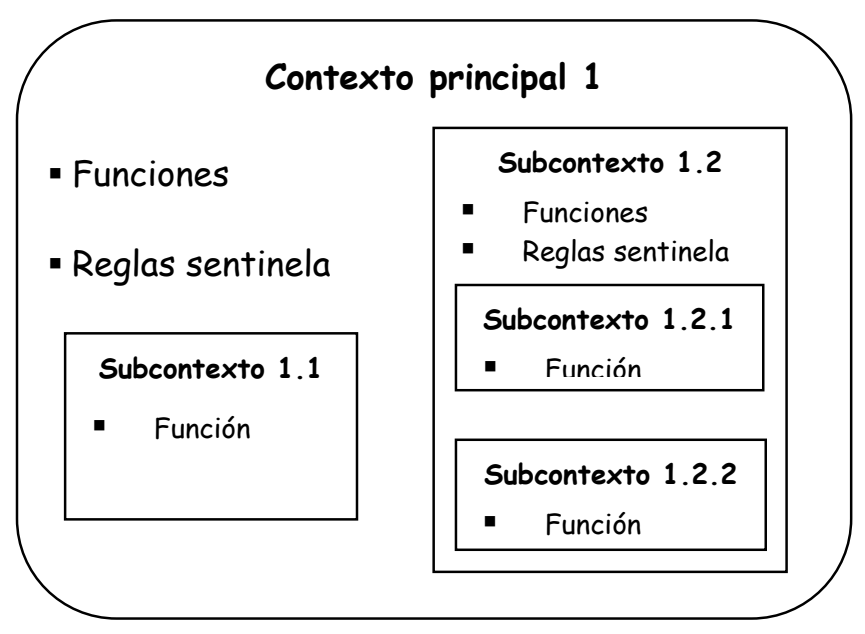

Figura 4 - Ejemplo de composición de contextos y subcontextos con funciones y reglas sentinela

\section{$\underline{\text { Reglas sentinela }}$}

Una regla sentinela especifica las condiciones que deben darse y el contexto o subcontexto que debe activarse en el caso de que esas condiciones sean verdaderas. Por ejemplo, el modelo de comportamiento de conductores costarricenses posee un contexto principal llamado ciudad, dentro del cual se incorpora la siguiente regla sentinela:

"Si se llega a una entrada a la autopista en donde se observa una carretera amplia con varios carriles de circulación, con una división física (muro, árboles, etc) separando los sentidos de circulación, desactivar este contexto principal y activar el contexto principal autopista."
La regla sentinela anterior permite llevar a cabo la transición de un contexto principal (ciudad) a otro principal (autopista). Este tipo de reglas desactivan totalmente el contexto principal actual y activan el contexto especificado en la regla.

El otro tipo de reglas sentinela son las que permiten realizar la transición hacia un subcontexto del contexto actual. Siguiendo con el mismo ejemplo del contexto principal ciudad, la siguiente es una regla sentinela de este tipo:

Si el automóvil de adelante avanza lentamente (su velocidad es menor al $80 \%$ de la velocidad deseada), y el carril de la izquierda tiene el mismo sentido de circulación, activar el subcontexto adelantar-con-mismo-sentido.

Este tipo de reglas hacen que el control que posee el contexto actual sea transferido temporalmente al subcontexto, o sea, se activa el subcontexto. Una vez finalizado éste, el control retorna automáticamente al contexto anterior.

Obsérvese que sólo los contextos principales tienen reglas sentinela para realizar la transición a otros contextos del mismo nivel, además de las reglas sentinela para activar subcontextos. Por su parte, los subcontextos sólo tienen reglas sentinela para activar sub-subcontextos.

\section{Resultados}

El modelo de comportamiento de conductores de automóviles de Costa Rica, está conformado por un conjunto de contextos y subcontextos. Éstos, a su vez, están enmarcados por una misión que define un objetivo y un plan de ejecución. A continuación se describe la misión y el diseño de los contextos.

Misión

Contexto de la Misión:

Trasladarse de localidad.

Descripción:

Transportarse en automóvil de una localidad a otra en el área metropolitana de Costa Rica.

\section{Automóvil:}

Vehículos livianos de un cuarto a una y media tonelada, correspondientes a los autorizados para ser conducidos con licencia tipo B-1 [6].

Clima:

Seco. 
Iluminación:

Luz del día.

Lugar de inicio:

Sede Rodrigo Facio de la Universidad de

Costa Rica (UCR), San José, Costa Rica.

Destino meta:

Aeropuerto Internacional Juan Santamaría (AIJS), Costa Rica.

\section{Objetivo:}

Llegar al lugar destino tan pronto como sea posible, en forma segura y sin daño al automóvil, en forma representativa a un chofer costarricense con las siguientes características:

1. Hombre

2. Entre 35 y 45 años de edad

3. Con un mínimo de experiencia de 5 años en manejo de automóviles

Restricciones de la misión:

1. Leyes de tránsito: constituyen una restricción hasta el punto donde los conductores costarricenses obedezcan las leyes.

2. Funcionamiento del automóvil.

3. Evasión de contacto físico con objetos estáticos o en movimiento.

4. Estado de las calles.

5. Supervivencia.

Secuencia planeada de contextos principales (ver figura 5):

1. Parqueo: (Campus universitario de la Sede Central de la UCR)

- Transición: salida del campus universitario por la Facultad de Derecho.

2. Pista: (Circunvalación San Pedro-Guadalupe)

- Transición: semáforos al final de la pista.

3. Ciudad: (Uruca)

- Transición: semáforos en la entrada a la autopista.

4. Autopista: (Bernardo Soto)

- Transición: entrada al aeropuerto.

5. Parqueo: (Aeropuerto Internacional J.S.)

- Transición: espacio de estacionamiento.

Contextos reactivos principales:

1. Reparar-carro

2. Llenar-tanque

\section{Contextos principales}

1. Parqueo

2. Pista
3. Ciudad

4. Autopista

Contextos principales reactivos

1. Reparar-carro

2. Llenar-tanque

$\underline{\text { Subcontextos }}$

1. Adelantar-con-mismo-sentido

2. Adelantar-con-sentido-contrario

3. Cruzar-zona-escolar

4. Cruzar-zona-peatonal

5. Evitar-colisión-emergencia

6. Evitar-hueco

7. Evitar-obstáculo

8. Evitar-peatón-animal

9. Evitar-presa

10. Evitar-vehículo

11. Giro-derecho

12. Giro-izquierdo

13. Oficial-de-tránsito

14. Pasar-peaje

15. Puesto-control-parqueo

16. Rotonda

17. Salida-carretera

18. Semáforo

19. Señal-tránsito

20. Vehículo-emergencia

Estructura del modelo de comportamiento de conductores

El modelo de tipo CxBR puede considerarse como una composición de contextos y subcontextos, algunos de los cuales son componentes de varios contextos a la vez. Sin embargo, dependiendo del contexto al cual pertenezca un subcontexto, los valores de algunas variables cambian.

A nivel más general, se presenta la jerarquía de contextos principales en la figura 6. Luego para cada uno de estos contextos, incluyendo los reactivos, se muestra una figura (de la 7 a la 12) mostrando su estructura.

Cada contexto principal está compuesto de un conjunto de funciones y de un grupo de reglas sentinela. Cada regla sentinela activa un subcontexto $\mathrm{u}$ otro contexto principal. Dado que algunos subcontextos y funciones son parte de varios contextos principales a la vez, para aplicar la reutilización se han parametrizado las funciones y las reglas sentinela que activan los subcontextos. 


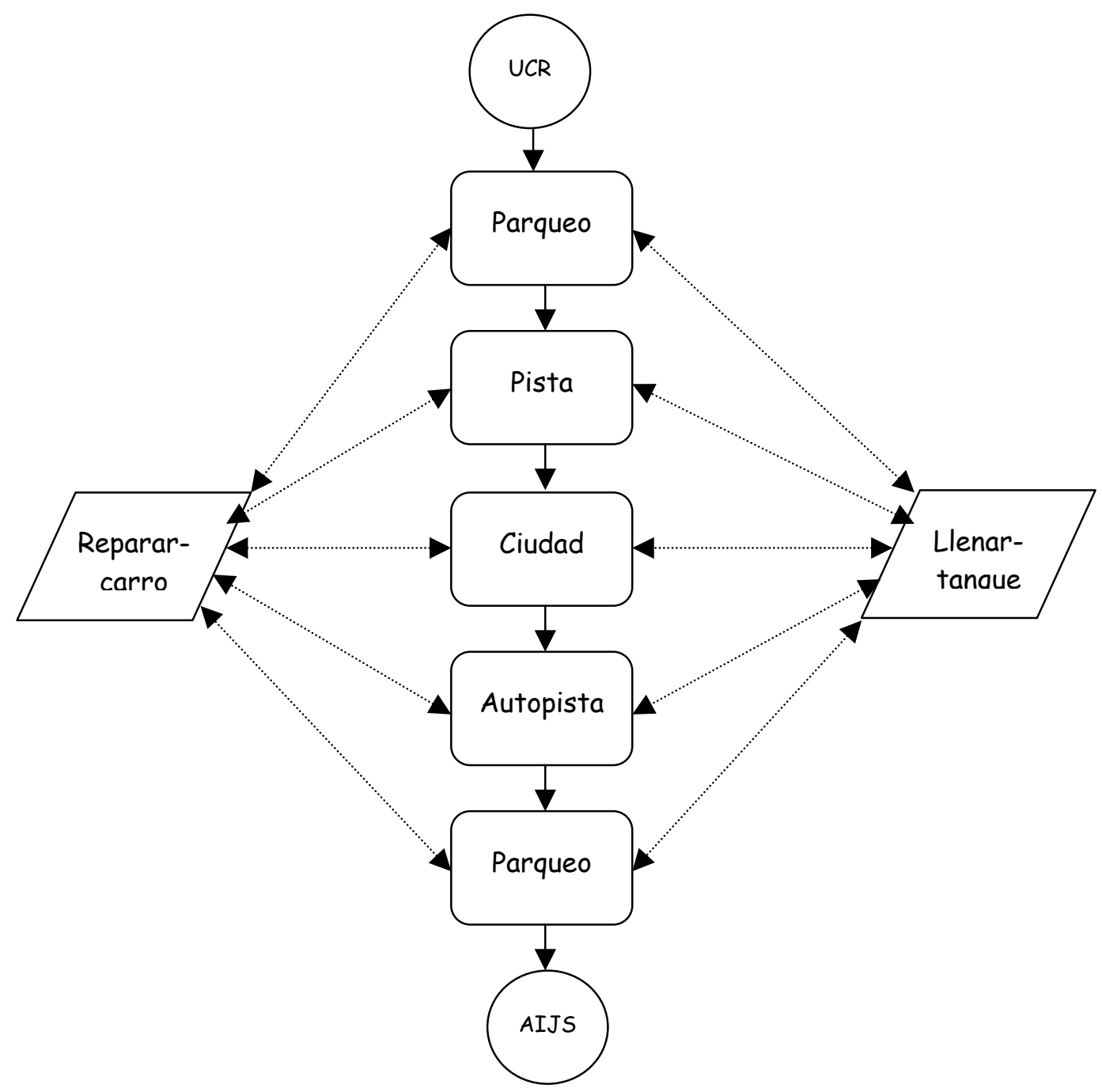

Figura 5 - Planificación de la misión del modelo de comportamiento de conductores de automóviles en Costa Rica

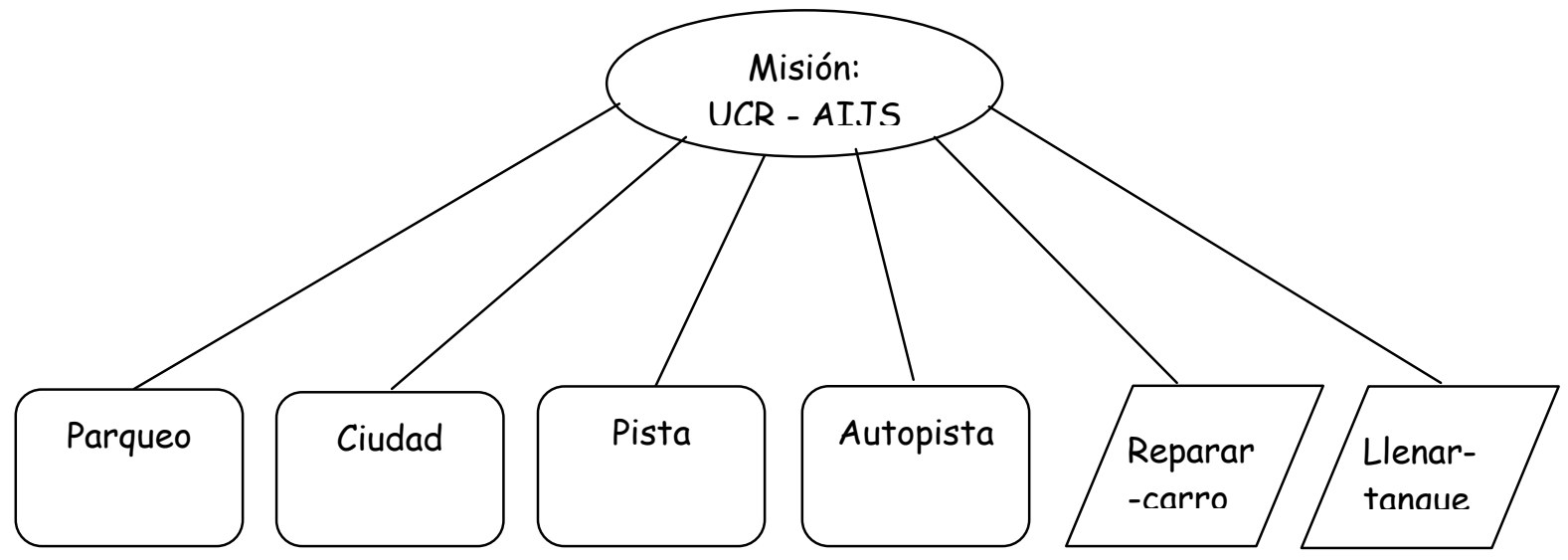

Figura 6 - Jerarquía de contextos principales del modelo de comportamiento de conductores de automóviles en Costa Rica 


\section{Parqueo}

- Funciones:

1. Avanzar (velActual, $25 \mathrm{~km} / \mathrm{h}$, null, $10 \mathrm{~km} / \mathrm{h}$ ).

2. Retroceder (velActual, $10 \mathrm{~km} / \mathrm{h}, 10 \mathrm{~km} / \mathrm{h}$ ).

3. Acelerar (velAcel, velMeta).

4. Desacelerar (velAcel, velMeta).

5. Detener.

6. Virar.

7. Dar-paso-vehículo-saliendo.

8. Salir-espacio-parqueo.

9. Vigilar-fallas.

- Reglas sentinela:

\section{Subcontextos:}

1. Evitar-vehículo

2. Evitar-peatón-animal

3. Evitar-colisión-emergencia

4. Evitar-obstáculo

5. Evitar-hueco

6. Señal-tránsito

7. Giro-derecho

8. Giro-izquierdo

9. Cruzar-zona-peatonal

10. Puesto-control-parqueo

1. Evitar-vehículo (90 grados, 10 metros, 1 metro).

2. Evitar-peatón-animal ( 90 grados, 10 metros, 1 metro).

3. Evitar-colisión-emergencia (3 segundos, 3 metros).

4. Evitar-obstáculo (25 metros).

5. Evitar-hueco (5 metros).

6. Señal-tránsito (25 metros).

7. Giro-derecho.

8. Giro-izquierdo.

9. Cruzar-zona-peatonal (10 metros).

10. Puesto-control-parqueo (5 metros).

11. Reparar-automóvil.

12. Llenar-tanque-combustible.

13. Entrar-a-ciudad.

14. Entrar-a-bista.

Figura 7 - Composición del contexto principal "Parqueo" 


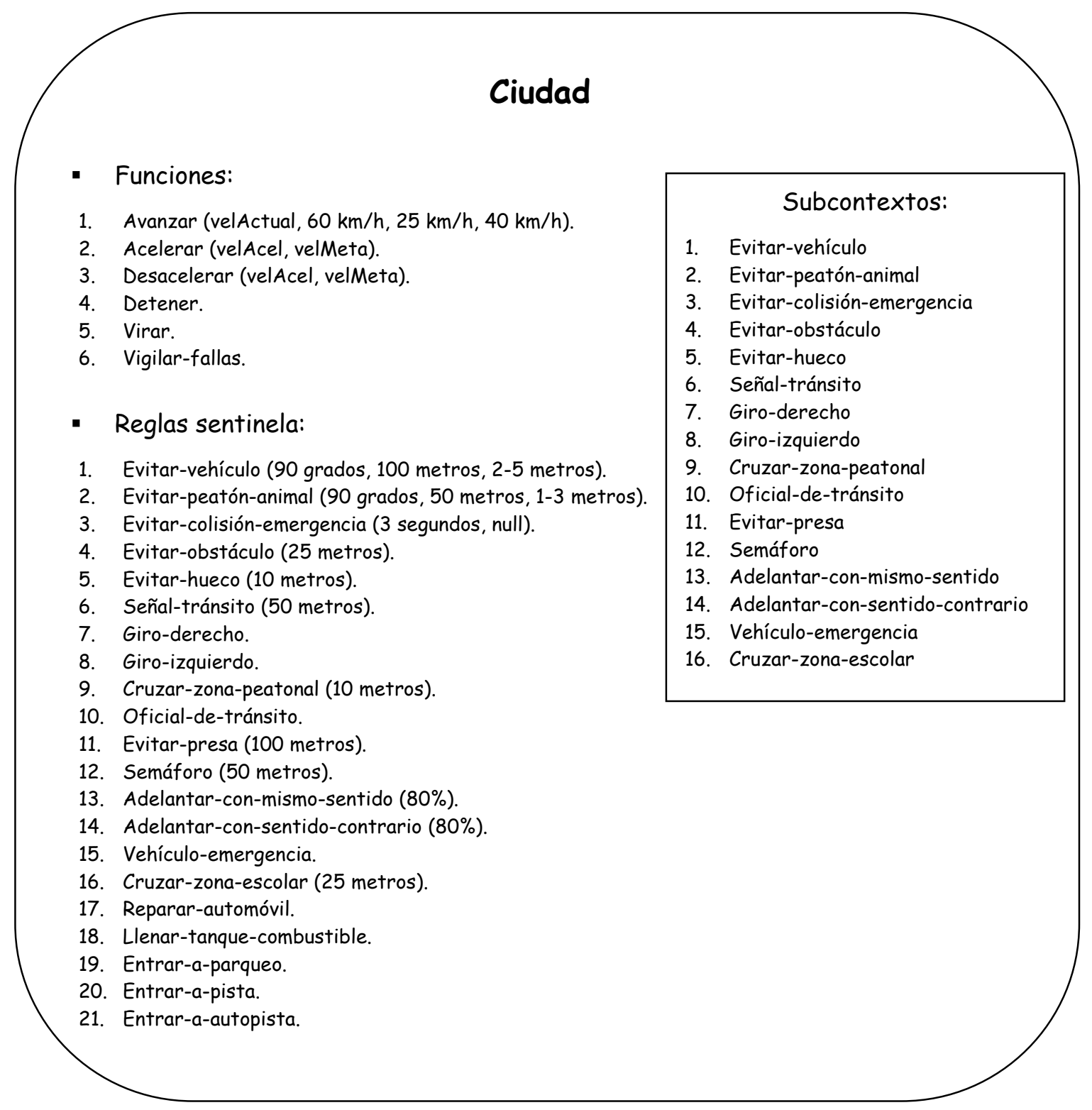

Figura 8 - Composición del contexto principal "Ciudad" 


\section{Pista}

- Funciones:

1. Avanzar (velActual, $80 \mathrm{~km} / \mathrm{h}, 40 \mathrm{~km} / \mathrm{h}, 65 \mathrm{~km} / \mathrm{h}$ ).

2. Acelerar (velAcel, velMeta).

3. Desacelerar (velAcel, velMeta).

4. Detener.

5. Virar.

6. Vigilar-adelantamientos (4 metros).

7. Vigilar-entradas (50 metros).

8. Vigilar-fallas.

- Reglas sentinela:

1. Evitar-peatón-animal (45 grados, 50 metros, 3 metros).

2. Evitar-colisión-emergencia (3 segundos, null).

3. Evitar-obstáculo (100 metros).

4. Evitar-hueco (25 metros).

5. Salida-carretera (50 metros).

6. Oficial-de-tránsito.

7. Evitar-presa (100 metros).

8. Semáforo (75 metros).

9. Rotonda (100 metros).

10. Adelantar-con-mismo-sentido ( $90 \%)$.

11. Vehículo-emergencia.

12. Reparar-automóvil.

13. Llenar-tanque-combustible.

14. Entrar-a-parqueo.

15. Entrar-a-ciudad.

16. Entrar-a-autopista.

\section{Subcontextos:}

1. Evitar-peatón-animal

2. Evitar-colisión-emergencia

3. Evitar-obstáculo

4. Evitar-hueco

5. Salida-pista

6. Oficial-de-tránsito

7. Evitar-presa

8. Semáforo

9. Rotonda

10. Adelantar-con-mismo-sentido

11. Vehículo-emergencia

Figura 9 - Composición del contexto principal "Pista" 


\section{Contexto principal "Autopista"}

\section{Autopista}

\section{- Funciones:}

1. Avanzar (velActual, $120 \mathrm{~km} / \mathrm{h}, 40 \mathrm{~km} / \mathrm{h}, 90 \mathrm{~km} / \mathrm{h}$ ).

2. Acelerar (velAcel, velMeta).

3. Desacelerar (velAcel, velMeta).

4. Detener.

5. Virar.

6. Vigilar-adelantamientos (15 metros).

7. Vigilar-entradas (50 metros).

8. Vigilar-fallas.

- Reglas sentinela:

1. Evitar-peatón-animal (45 grados, 50 metros, 3 metros).

2. Evitar-colisión-emergencia (3 segundos, null).

3. Evitar-hueco (25 metros).

4. Salida-carretera (50 metros).

5. Evitar-presa (100 metros).

6. Adelantar-con-mismo-sentido ( $90 \%)$.

7. Vehículo-emergencia.

8. Oficial-de-tránsito.

9. Pasar-peaje (100 metros).

10. Reparar-automóvil.

11. Llenar-tanque-combustible.

12. Entrar-a-parqueo.

13. Entrar-a-ciudad.

14. Entrar-a-pista.

\section{Subcontextos:}

12. Evitar-peatón-animal

13. Evitar-colisión-emergencia

14. Evitar-hueco

15. Salida-pista

16. Evitar-presa

17. Adelantar-con-mismo-sentido

18. Vehículo-emergencia

19. Oficial-de-tránsito

20. Pasar-peaje

Figura 10 - Composición del contexto principal "Autopista" 


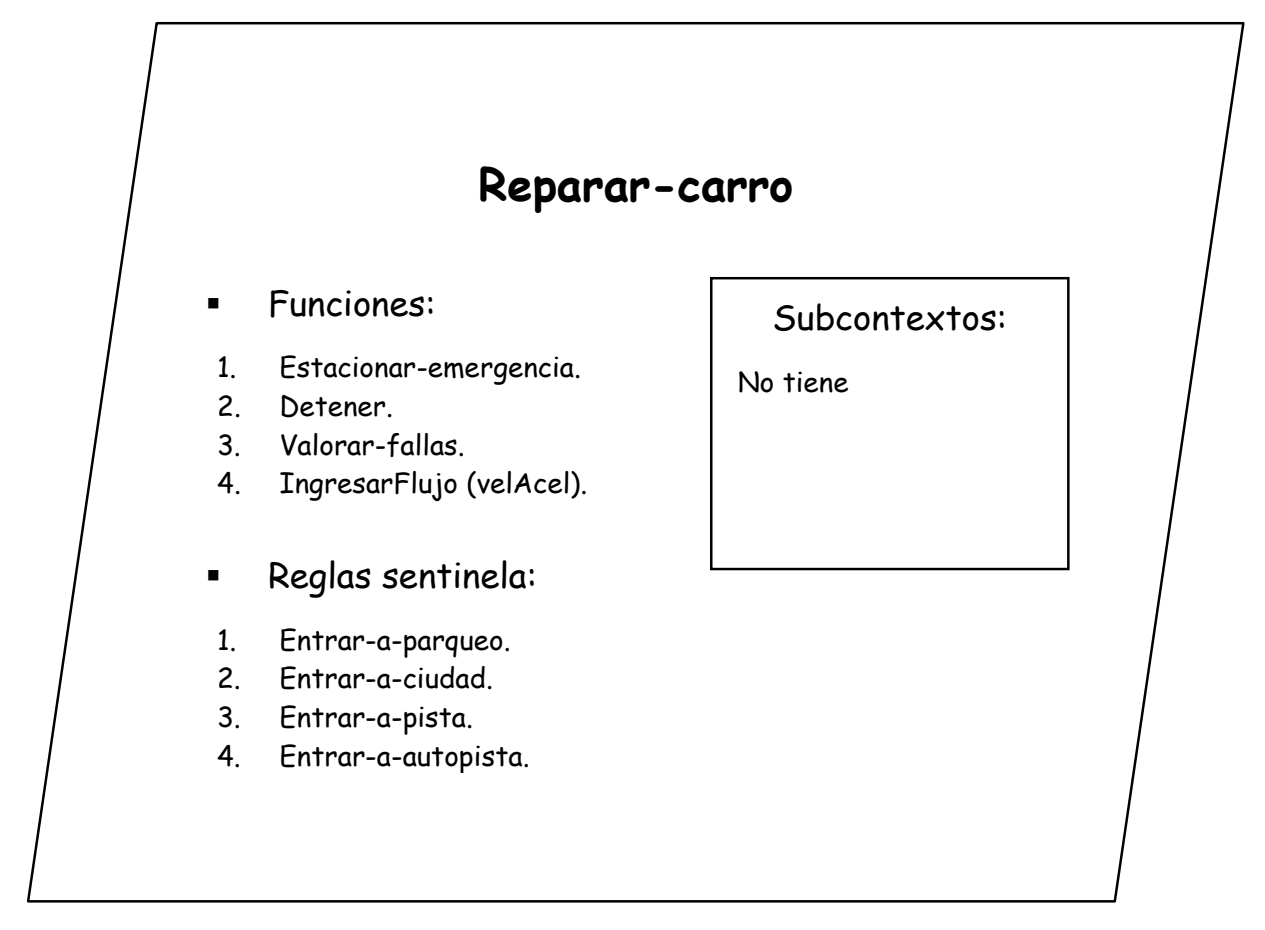

Figura 11 - Composición del contexto principal reactivo "Reparar-carro" 


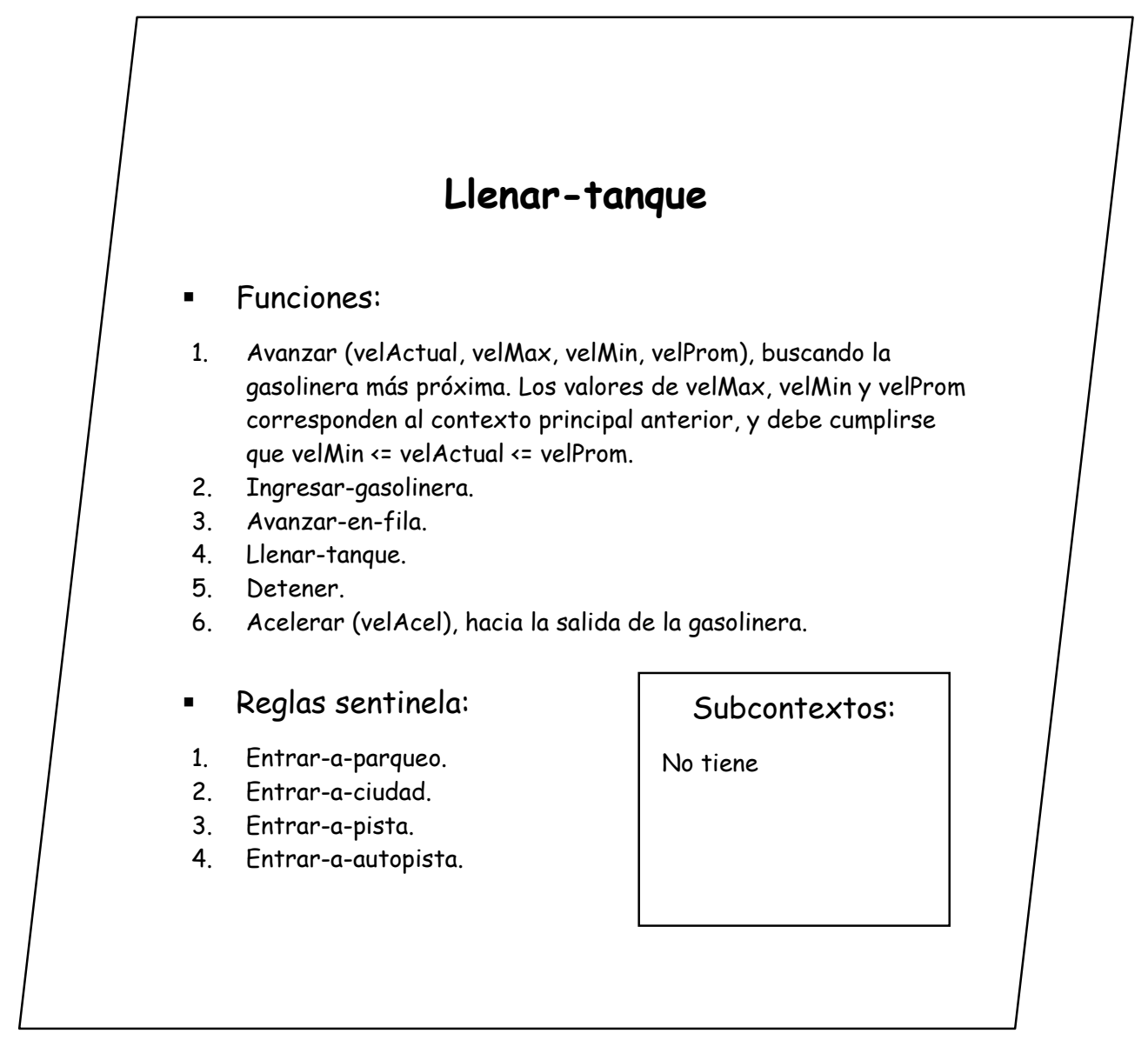

Figura 12 - Composición del contexto principal reactivo "Llenar-tanque"

\section{Conclusiones}

Luego de desarrollar el modelo teórico de comportamiento de conductores de automóviles de Costa Rica y de compararlo con la especificación de un modelo similar para conductores de Estados Unidos [7], se observa que las funciones de ambos modelos son similares. Su principal diferencia son los valores que asumen algunas variables como distancia y velocidad. Sin embargo, los contextos del modelo costarricense son más amplios en cuanto al conocimiento que deben incluir, ya que las circunstancias a las que se enfrenta un conductor en diferentes contextos son similares entre sí. La frontera del comportamiento entre los contextos no es tan definida como en el modelo estadounidense. Por lo tanto, el conocimiento de cada contexto debe cubrir un número mayor de situaciones.

Para facilitar la creación de modelos de razonamiento basados en contextos, debe promoverse el desarrollo de unidades $u$ objetos reutilizables. Esto favorece la implementación de modelos cuyos contextos abarcan funciones, reglas sentinela y subcontextos comunes, tal como el modelo descrito. También permite aprovechar el trabajo y experiencia de desarrolladores que han creado modelos similares.

El paradigma CxBR requiere de una organización, generalmente jerárquica, de las bases de conocimiento. Los contextos permiten estructurar el conocimiento en unidades reutilizables, definiendo el momento en que cada unidad se activa. De esta forma, el razonamiento basado en contextos cobra mayor importancia en problemas en que se manipula gran cantidad de conocimiento, no necesariamente especializado, tal como el caso de sistemas que intentan simular la intuición humana aplicada a situaciones del diario vivir.

La organización del conocimiento en unidades que se realiza con este paradigma de modelaje de comportamiento humano, permite gran flexibilidad 
en el caso de contextos en que el conocimiento es variable, ya que facilita el eliminar o integrar nuevas situaciones a una misión. Por ejemplo, con respecto al modelo presentado en este artículo, podría eventualmente ser innecesario el paso por rotondas para alcanzar el objetivo, dada la tendencia de las autoridades a realizar cambios viales en carreteras.

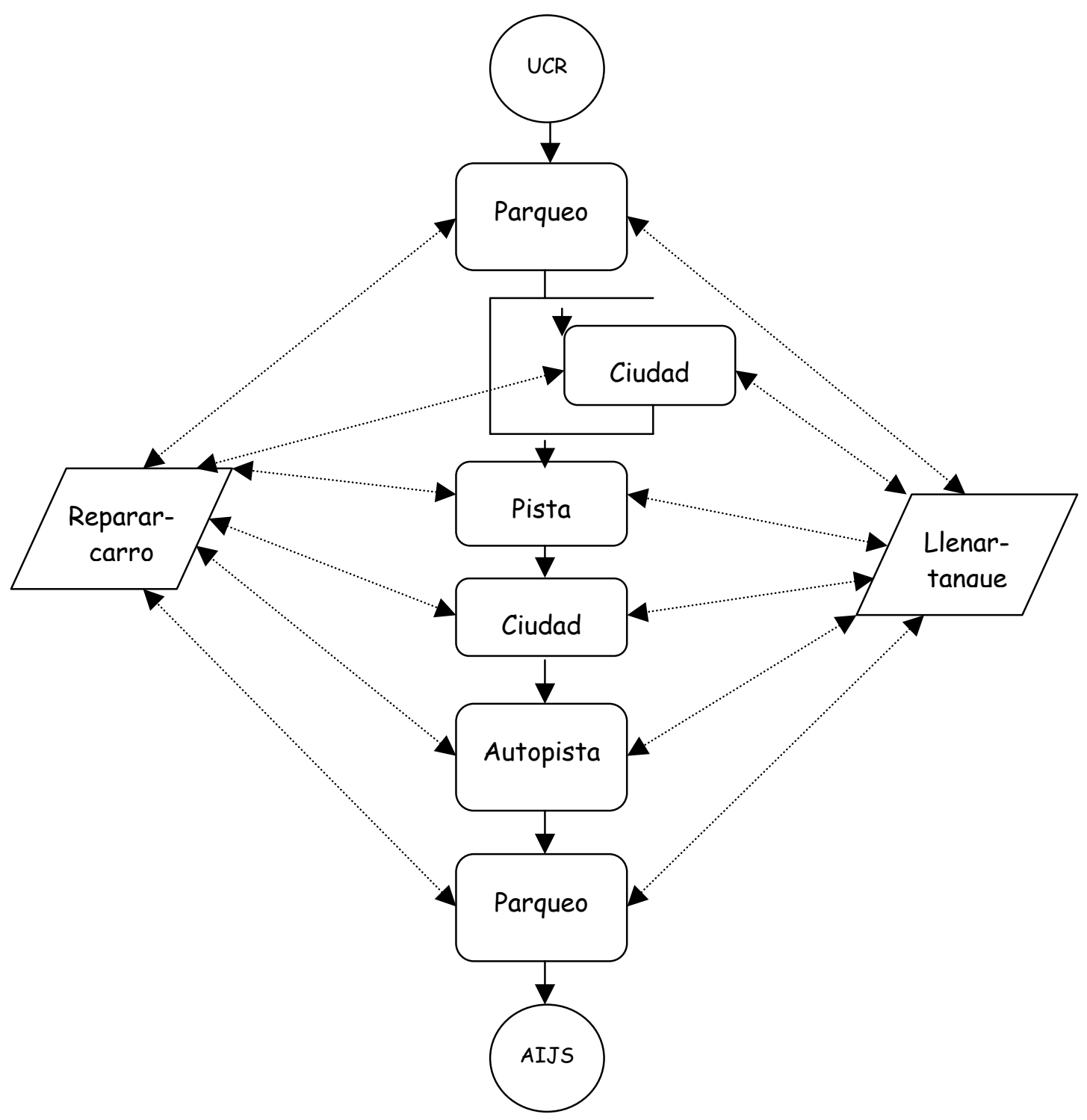

Figura 13 - Ejemplo de planificación alterna para la misión del modelo de comportamiento de conductores de automóviles en Costa Rica

En una misión como la presentada, típicamente se define una secuencia de contextos, lo que constituye una planificación de contextos a activar. También se definen contextos reactivos que se activan ante situaciones que se presentan súbitamente y que podrían no darse del todo. Sin embargo, un modelo podría definir planes alternos cuyo seguimiento no estaría predeterminado al inicio de la misión, sino durante su desarrollo dependiendo de las circunstancias ante las que se enfrente el agente inteligente en un momento dado.

Por ejemplo, dependiendo de cuál salida del parqueo se tome, podría ingresarse a la ciudad primero para luego llegar a la pista, o podría ingresarse directamente a la pista (ver figura 13). La 
salida del parqueo que se tome no puede definirse de antemano al plantear la misión, dependerá del espacio en se encuentre estacionado inicialmente el vehículo, de posibles salidas cerradas, o de presas dentro del parqueo.

\section{Agradecimentos}

Se agradecen los aportes del Dr. Avelino González, investigador y profesor de University of Central Florida, Estados Unidos, y asesor del proyecto del cual se derivan los resultados expuestos en este documento; al Dr. José Ronald Argûello, investigador y profesor de la Universidad de Costa Rica, Costa Rica, y asesor de este proyecto.

\section{Referencias}

1. González, Fernando; Patric, Grejs; González, Avelino. Autonomous Automobile Behavior Through Context-based Reasoning. Florida Artificial Intelligence Research Society Conference. Estados Unidos (2000)

2. Saeki, Shynia; González, Avelino. Soft-coding the Transitions Between Contexts in CGF's: the Competing Context Concept. Conference:
Computer Generated Forces and Behavior Representation (2000)

3. González, Avelino; Ahlers, Robert. A Contextbased Representation of Tactical Knowledge for Use in Simulator-based Autonomous Intelligent Platforms. Proceedings of the 15th Annual Interservice/Industry Training Systems and Education Conference. Orlando, Florida, United States (1993) 543-552

4. González, Avelino; Ahlers, Robert. Contextbased Representation of Intelligent Behavior in Simulated Opponents. Conference: Computer Generated Forces and Behavior Representation (1995)

5. González, Avelino; Ahlers, Robert. Contextbased Representation of Intelligent Behavior in Training Simulation. Conference: Naval Air Warfare Center Training Systems Division (1998)

6. Consejo de Seguridad Vial. 20 Años de Estadísticas de Accidentes de Tránsito, Costa Rica 1981-2000. Costa Rica (2000)

7. González, Avelino. Specification of Automobile Driving Model. Intelligent Systems Laboratory. University of Central Florida. Orlando, Florida, United States (1998) 